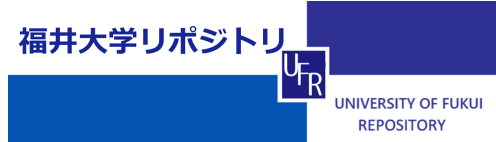

Rot at i onal di ffusi on current of mul ti-char ged redox speci es by means of Monte Carlo si mil at i on

\begin{tabular}{|l|l|}
\hline $\begin{array}{l}\text { j our nal or } \\
\text { publ i cat } \mathrm{i} \text { on } \mathrm{t} \text { i t l e }\end{array}$ & El ect r ochi mi ca Act a \\
\hline vol une & 51 \\
\hline number & 27 \\
\hline page $\mathrm{r}$ ange & $6012-6017$ \\
\hline year & $2006-08$ \\
\hline URL & ht t p: //hdl . handl e. net /10098/1149 \\
\hline
\end{tabular}


Grotthuss-015

\title{
Rotational diffusion current of multi-charged redox species by means of Monte Carlo simulation
}

\author{
Koichi Aoki ${ }^{*}$ \\ Department of Applied Physics, University of Fukui, 3-9-1 Bunkyo, Fukui-shi, 910-8507 \\ Japan,
}

\begin{abstract}
Multi-charged redox species such as dispersed metal nanoparticles and latex particles have exhibited diffusion-controlled currents, which may be caused by not only the conventionally translational diffusion but also by the rotation of the species or random orientation toward the electrode. Rotational diffusion was here taken into account by use of the multiple redox-charged model particle which moves back and force in thin layer cell as well as rotates clockwise and counterclockwise by means of the Monte Carlo simulation. The steady-state current was smaller than the current which might flow if all the charge were to transfer at infinite rotational speed. The current values had a linear relation with the $-1 / 2$ powers of the number of the charge per particle. This relation was in accord with the experimental results of the partial charge transfer reaction of the ferrocene-immobilized latex suspension.
\end{abstract}

key words: Monte Carlo simulation, diffusion current, rotation, nanoparticles, latex particles

\footnotetext{
*d930099@icpc00.icpc.fukui-u.ac.jp, phone +81 77627 8665, fax +81 776278494 


\section{Introduction}

Metal complexes with multiple charge transfer reactions were classically explored at the aim of creating new redox systems by taking advantages of formation of mixed-valence states through $\pi$-conjugation [1-3]. Electrochemical techniques have played a role in estimating the mixed-valence states [4-6]. The voltammetric behavior of mixed-valence states has been theoretically developed in the context of combinations of pair potentials between redox sites [7-10], and has allowed us to evaluate quantitatively interaction energy of the mixed-valence states [11-13]. Multi-charge redox species can also be seen in redox polymers which are coated on electrodes [14-17]. Their electrochemical behavior is actually the same as that of the monomer, because each redox site in the polymer reacts independently $[15,16]$. Recently attractive multi-charged redox specie are metal nanoparticles [18-21], clusters [22], dendrimers [23,24], fullerenes, redox proteins, redox polyelectrolytes [25-29], and redox colloids. Since they have macroscopic size, geometry and a clear boundary from solvent, they may have properties of particles, discriminated against polymer-coated electrodes.

These jumbo redox particles are predicted to exhibit complicated electrode reactions in current-potential curves. The current-potential curves for $n$ redox sites each with a one-electron transfer reaction show two and three waves for even and odd numbers of $n$, respectively $[7,8]$ when the redox pairs are stabilized. If pair interactions are communicated each other complicatedly, $n$ waves appears [10]. When $n$ reactions occurs consecutively without any interaction among redox sites, only one wave appears [30], of which waveform is identical with the wave of the Nerstian one-electron reaction. The presence of a simple interaction makes the formal potential be shifted with $1 / n$ dependence [31-33].

When jumbo redox particles are adsorbed on an electrode, the Faradaic current has less information of properties of particles than voltammetric potentials because it varies with amount of aggregation. In contrast, diffusion-controlled currents are expected to include properties of particles such as size, concentration, the number of charge per particle. They have not been examined yet unfortunately, because of geometrical complications such as contact area of the particle with an electrode, charge exchange reaction between nearest redox 
sites within a particle, rotation of the particle, effects of the double layer on the charge transfer. The voltammetric diffusion-controlled peak currents for $n$-electron particles has been estimated intuitively to be proportional to $n$ [34] rather than the conventional proportionality to $n^{3 / 2}$ [35-38]. The $n$-proportionality has been demonstrated theoretically by use of the model of the consecutive reaction [30]. Size-dependence of the diffusion currents has been investigated by use of ferrocenyl latex particles. Particles smaller than $0.1 \mu \mathrm{m}$ in diameter $[39,40]$ show conventional diffusion-controlled current, whereas those larger than $0.5 \mu \mathrm{m}$ $[41,42]$ show currents smaller than the diffusion-controlled current. The observed size-dependence may suggest a contribution of the rotational diffusion.

We consider as an example of the rotational diffusion a model of ferrocene-coated latex particles suspended in a solution. The ferrocenyl moieties in a particle are assumed to have no redox exchange reaction among the moieties. When a particle collides with the electrode, ferrocenyl moieties only within the electric double layer can be oxidized at the electrode, the other moieties being still under the reduced state. Random force acting on the particle causes not only translation but also rotation. The translation near the electrode makes the particle collide with the electrode to yield the current. In contrast, the rotation does not contribute directly to the current except for on the electrode. Therefore the current is predicted to be smaller than the conventional diffusion-controlled current. This diffusion process including both translation and rotation is carried out by means of the Monte Carlo simulation in this report.

\section{Model}

\subsection{Reaction system}

The model system proposed is a diffusing circular particle, $a$ in radius, with $n$ common redox sites, at each of which the redox reaction, $\mathrm{R} \leftrightarrow \mathrm{O}+\mathrm{e}^{-}$, occurs. The $n$ redox charges are distributed on the surface of the circle at a uniform density without any interaction between the redox charges. The only one particle is located in a rectangular cell $w$ wide with an anode and a cathode, as is illustrated in Fig.1. The anode and the cathode are kept to be sufficiently 
anodic and cathodic potentials, respectively, so that the reaction is controlled by mass transport.

The cell contains supporting electrolyte with high enough concentration for neglecting the electric migration. If $n$ redox charges were to be distributed uniformly over the cell, as would be predicted for dissolution of the redox latex particle, the one-dimensional average concentration would be ne/w. Then the concentration distribution at the steady-state electrolysis should be proportional to the distance, $x$, from the cathode, to give $(n e / w)(x / w)$. The current density for the uniform distribution is then given by

$$
j_{\text {unf }}=(n e / w) D / w
$$

This is essentially the same as the expression for the current density in a thin layer cell, $j=n F C^{*} D / w$.

If the $n$ electrons on the particle were to be condensed as a point charge, the time-averaged concentration should be uniform over the cell because of random walk of the particle. Consequently, Eq.(1) holds even for the condensed charge for a long time.

Collision of the particle with solvent molecules causes random walk to yield diffusion. We shall evaluate the current by means of the Monte Carlo simulation not only by translating the particle randomly toward either the cathode or the anode but also by rotating the particle either clockwise or counterclockwise. When a force, $f$, acts on a surface point of the particle (Fig.1), its $x$-components, $f_{x}$, drives the particle to yield the $x$-component of the velocity, $v_{x}$, whereas the angular component, $f_{\theta}$, rotates the particle to give the angular velocity, $\omega$, or the circular velocity, $v_{\theta}=a \omega$. The force, $f_{x}$, is balanced with the frictional force, $6 \pi \eta a v_{x}$, against the Newtonian fluid in the viscosity, $\eta$, according to the Stokes law [43]. On the other hand, the torque, $a f_{\theta}$, is balanced with the rotationally frictional torque, $8 \pi \eta a^{3} \omega\left(=8 \pi \eta a^{2} v_{\theta}\right)$ [44]. If force, $f$, being ascribed to the thermal fluctuation energy $k_{\mathrm{B}} T$ for the displacement of $\Delta x$, causes both the translation and the rotation, the two velocities are given by

$v_{\mathrm{x}}=f / 6 \pi \eta a=k_{B} T / 6 \pi \eta a \Delta x$

$v_{\theta}=f / 8 \pi \eta a=k_{B} T / 8 \pi \eta a \Delta x$

where $k_{\mathrm{B}}$ is Boltzmann's constant. Dividing both sides by $\Delta x$, we obtain the definition of the diffusion coefficients for the translation and the rotation: 
$v_{\mathrm{x}} \Delta x=k_{\mathrm{B}} T / 6 \pi \eta a \equiv D_{\mathrm{x}}$

$v_{\theta} \Delta x=k_{\mathrm{B}} T / 8 \pi \eta a \equiv D_{\theta}$

The rotational diffusion coefficient is $3 / 4$ times of the translational diffusion coefficient.

\subsection{Monte Carlo simulation}

We assume that the translation is limited to the $x$-direction and that the rotation is restricted to the $\theta$-direction (Fig.1). The particle translates back and force by the digitized value, $\pm \Delta x$, randomly during the period, $\Delta t$, for each of which the random force acts on the particle. The center of the particle is necessarily located at a multiple of $\pm \Delta x$ on the $x$ axis, and hence we digitize the diffusion space (length), $w$, into $N$ so that $w=N \Delta x$. In contrast, the particle rotates by the arc length, $\Delta x$, or by the radian, $\Delta x / a(=\Delta \theta)$ in $\Delta t$. In order to keep the rotational symmetry, we digitize the circumference of the particle by $\Delta x$ so that

$2 \pi a / \Delta x=n$

where $n$ is an even number. In other word, the particle is approximated as a regular polygon with an even number of sides (see the octagon for $2 \pi a / \Delta x=8$ in Fig. 1). One side of the polygon has either the elementary charge $e$ or the vacancy, and hence the most highly reduced particle possesses ne charge. When a side of the polygon comes in contact with either the anode or the cathode after the translation or the rotation, the transfer reaction occurs.

The left and the right side of the polygon can collide with the anode and the cathode, respectively, as is indicated by bold lines on the octagon in Fig.1. The distance between the colliding site and the center, $a \cos (\Delta x / a)$, is not a multiple of $\Delta x$. Consequently the both electrodes are shifted to the nearest lattice points, as illustrated in the bold arrows of Fig.1. When the left side with the reduced moiety collides with the anode, it is oxidized to release the charge $e$. Nothing happens if the left side with the oxidized moiety collides with the anode. The number of collisions associated with the charge transfer is counted and is defined as $j \Delta t / e$. The number divided by the number of dimensionless time or the number of generating random numbers is the dimensionless current.

The whole process in the Monte Carlo simulation includes in order

(i) setting values of $N$ and $w / a$ so that $n=2 \pi N a / w$ is an even number,

(ii) relocating the anode at a rounded value of $n / 2 \pi$, and the cathode at $N-n / 2 \pi$, 
(iii) initializing the location of the center or $n$ redox sites, and $n$ redox states $(\mathrm{O}$ or $\mathrm{R})$,

(iv) generating a random number ranging 0 to 1 as a selection of the translation or the rotation,

(v) generating a random number, $\mathrm{r}$, for the translation of $\Delta x$ for $0.5<\mathrm{r}$ or $-\Delta x$ for $\mathrm{r}<0.5$ (or rotation of $\Delta \theta$ for $0.5<\mathrm{r}$ or $-\Delta \theta$ for $\mathrm{r}<0.5)$,

(vi) examining the collision of the left or the right side of the polygon with the electrode

(vii) going back to step (iv) until the current becomes a steady state.

The probability of whether the translation or the rotation occurs is proportional to the value of the diffusion coefficient, because the force acting on the particle is proportional to $k_{\mathrm{B}} T$ and thus to $D$ through Eq. (2) and (3). Then Eq.(2) and (3) give the probability of the translation:

$$
p_{\mathrm{x}} \leq D_{\mathrm{x}} /\left(D_{\mathrm{x}}+D_{\theta}\right)=(1 / 6) /[(1 / 6)+(1 / 8)]=4 / 7 \equiv \mathrm{R}_{\mathrm{th}}
$$

where $\mathrm{R}_{\mathrm{th}}$ is the threshold value under which the translation is predominant to the rotation.

The steps (iv)-(vii) are shown in the flow chart in Fig. 2. An example is given below. Let a hexagon be located at $\Delta x$ from the anode (see the right of Fig. 2). The hexagon has initially the oxidized moiety only on the right side (denoted by a dot), and the reduced moieties on the other five sides. If a random number for the threshold is 0.3 , the translation occurs. The next random number, 0.1 , moves the hexagon by $-\Delta x$ to make it collide with the anode. Since the colliding side is under the reduced state, it changes into the oxidized state by the transfer of the dimensionless charge. When the third and the forth random numbers are 0.8 and 0.7 , respectively, the hexagon rotates by $\Delta \theta$. It gives rises to the rotational collision with the electrode. Since the colliding side has the reduced moiety, it changes into the oxidized one.

\section{Results and Discussion}

\subsection{Justification of the Monte Carlo Method}

We shall justify the technique of the simulation and estimate the accuracy when $n$ electrons are condensed as a point charge. This condition corresponds to the simultaneous $n$-electron transfer at one collision without the rotation of the particle. Applying the definition of the diffusion coefficient, $D_{x}=(\Delta x)^{2} / 2 \Delta t$, to the analytical expression (Eq.(1)) for the 
present case , we rewrite Eq.(1) as

$$
j_{\text {unf }}(\Delta t) / e=(n / 2)(\Delta x / w)^{2}
$$

We carried out the Monte Carlo simulation at several values of $\Delta x / w$ by setting $n$ to be unity without any rotation. The dimensionless current, $j(\Delta t) / e$, was averaged with the number of generated random numbers. The square-roots of the average values are plotted against $1 / N(=$ $\Delta x / w)$ in Fig.3. They are proportional to $1 / N$, and the slope is very close to the theoretical (solid) line with $2^{-1 / 2}$. The present Monte Carlo simulation includes errors less than $3.5 \%$. The error increased with a decrease in $N$.

\subsection{Effects of rotational diffusion}

The Monte Carlo simulation associated with the rotation was carried out for various combinations of parameters of $w / 2 a$ (or $n$ ) and $N$. A trajectory of an indicated side of the 80-polygon is shown in Fig.4. It exhibits an ordered pattern of circles, which may be unexpected for the random motion. Arcs of the trajectory result from several digitized rotations around a given $x$. Although the trajectory is rather ordered, the charge transfer occurred almost randomly.

Figure 5 shows the variation of $[j(\Delta t) / e]^{1 / 2}$ with $1 / N$ for several values of $n$. Values of $[j(\Delta t) / e]^{1 / 2}$ for $n \leq 8$ are proportional to $1 / N$, as is similar to Eq.(5). Figure 5 also shows dotted lines of Eq.(5) for $n=2$ and 40. All the simulated values of $[j(\Delta t) / e]^{1 / 2}$ except for $n=1$ are smaller than values predicted from Eq.(5), because the rotational diffusion has less contribution to the current than the translational diffusion. As an example, the simulated slope at $n=2$ in Fig. 5 was 0.71 whereas the slope of the simultaneous reaction is unity from Eq.(5). That is, the ratio of the simulated current, $j_{\mathrm{sm}}$, to $j_{\text {unf }}$ for $n=2$ is $0.71^{2}=0.51$. The half contribution of the charge $(2 e)$ to the current can be explained as follows: When the particle possessing two reduced moieties collides with the anode (see Eq.(6), the anode being denoted A, (space) being vacancy), the redox site facing to the anode takes necessarily the oxidized form (Eq. (6-1)). Since the $4 / 7$ contribution of the random force causes the translational diffusion, the current is $(4 / 7) j_{\mathrm{sm}}$. The reduced moiety on the back side can be oxidized when it 
is rotated at the probability of $3 / 7$ (Eq. (6-1)). The probability of the second reduction is $(4 / 7)(3 / 7)$ because of requirements of both the translation and rotation. If the half-oxidized species translates $\Delta x$ and then $-\Delta x$, returning to the anode surface, the rotation causes the oxidation (Eq.(6-3)). Then the probability is $(4 / 7)^{2}(3 / 7)$.

$$
\begin{aligned}
& \mathrm{A}|(\quad)(\mathrm{RR}) \rightarrow \mathrm{A}|(\mathrm{OR})(\quad): \text { trnsltn } \\
& \mathrm{A}|(\quad)(\mathrm{RR}) \rightarrow \mathrm{A}|(\mathrm{OR})(\quad) \rightarrow \mathrm{A} \mid(\mathrm{OO})(\quad): \text { trnsltn, rttn } \\
& \mathrm{A}|(\quad)(\mathrm{RR}) \rightarrow \mathrm{A}|(\quad)(\quad)(\mathrm{RR}) \rightarrow \mathrm{A}|(\quad)(\mathrm{RR}) \rightarrow \mathrm{A}|(\mathrm{OR})(\quad): 3 \operatorname{trns} \mid \mathrm{tnn}, \mathrm{rttn}
\end{aligned}
$$

Therefore the ratio of the current up to three probabilities is given by

$$
j_{\mathrm{sm}} / j_{\mathrm{unf}} \approx\left(\frac{4}{7}+\frac{4}{7} \frac{3}{7}+\frac{4}{7} \frac{4}{7} \frac{3}{7}\right) / 2=0.48
$$

It is close to the simulated value $(0.51)$. There are some other minor processes.

With an increase in $n$, the variation of $[j(\Delta t) / e]^{1 / 2}$ with $1 / N$ deviates to a concave curve. Since the increase in $n$ and $1 / N$ enhances $a / w$ through $2 \pi a / w=n / N$, the rotational diffusion contributes largely to the current. The extreme case is that the two electrodes sandwich the particle, i.e., $2 a=w$. Then no translation is permitted and hence only the rotational diffusion can give rise to the redox reaction.

\subsection{Dependence of the current on charge number}

We examined the variation of the ratio, $j_{\mathrm{sm}} / j_{\text {unf }}$, with $n$ and $N$, and plotted the ratio against $n$ in the logarithmic scales for some values of $N$ in Fig.6. The ratio for $n<20$ does not vary with $n$. The invariant values are close to 0.5 , as is similar to Eq.(7). The first two terms in Eq.(7) are valid for the estimation of $j_{\mathrm{sm}} / j_{\text {unf }}$ for any value of $n$. Further increase in $n$ decreased the ratio for any value of $N$. The logarithmic plots showed linear variation with the slope of -0.5 . Consequently, the empirical relation is given by

$$
j_{\mathrm{sm}}=k^{\prime} j_{\mathrm{unf}} n^{-1 / 2}=k j_{\mathrm{unf}}(w / a N)^{1 / 2}
$$

where $k$ and $k^{\prime}$ are constants. The current is proportional to the inverse of the square-root of the radius. This relation expresses the size effect of the particle on the current. 
Two kinds of experimental data about the size effect are available: one being the diffusion-controlled voltammetric currents of the ferrocene-including polystyrene latex with the volumetrically uniform distribution [39], and the other being that of chemically immobilized ferrocenyl moieties on the surface of the polystyrene latex [40]. Since the concentration of the latex was low, there was no distribution of viscosity. The number of active redox charge, $n_{\mathrm{CV}}$, was evaluated from the current by use of the equation for the peak current of $n$-redox reactions [30]. This value was smaller than the number of the redox charge loaded on the particle, $n_{\mathrm{UV}}$, which was determined by the absorption of the UV-vis spectra of dissolved latex solution $[39,40,45]$. If $n_{\mathrm{UV}}$ is equal to $n$ (the number of the charge on the surface) in the present model, the ratio $n_{\mathrm{CV}} / n_{\mathrm{UV}}$ should be the same as $j_{\mathrm{sm}} / j_{\text {unf }}$. Figure 7 shows the plot of the ratio against the radii of the particles on the logarithmic scale for the two kinds of the ferrocene particles $[39,40]$. Both show linear variations. The ferrocene with the volumetrically uniform distribution [39] exhibits the slope -1 , whereas the surface-coated ferrocene has the slope -1.5 [40]. The former is not suitable to the present model in that the charge is present even inside of the particle, i.e., $n<n_{\mathrm{UV}}$, as is illustrated in Fig. 7. The latter agrees with the model in that all the redox charge is located on the latex surface owing to hydrophobic repulsion of the polyallylamine of the surface coating against the polystyrene core. The thickness of the ferrocenyl shell was proportional to the radius because of limitations of synthetic conditions $[40,42]$. Since $n_{\mathrm{UV}}$ is proportional to both the surface area and the thickness, it turns to be proportional to $a^{3}[40]$. Eliminating $a$ from the empirical slope, $j_{\mathrm{sm}} / j_{\mathrm{unf}}=k_{2} a^{-3 / 2}$, by use of $n_{\mathrm{UV}}=k_{1} a^{3}$, we obtain $j_{\mathrm{sm}} / j_{\mathrm{unf}}=k_{3} n_{\mathrm{UV}}{ }^{-1 / 2}$. This is the same result as the linear portion in Fig. 6.

We have assumed that the particle is a circle in the two-dimensional space although the two diffusion coefficients are for a sphere. In order to make the two models consistent, we attempted to use diffusion coefficients of a circle. Since diffusion coefficients of a circle are not available and not practical, we varied the threshold value from Eq. (4). When $R_{\text {th }}$ increased from 0.45 to 0.70 , values of $j_{\mathrm{sm}} / j_{\text {unf }}$ for $n \leq 14$ increased from 0.4 to 0.7 . Since the increase in $\mathrm{R}_{\text {th }}$ is favorable to the translational diffusion, the current enhances at the cost of the rotational contribution. When $n \geq 20$, the linear relation was still held and slope was -0.5 , 
independent of the threshold values. Therefore the difference between the two- and the three-dimension is not significant as for the -0.5-power dependence.

Concentration profiles in the diffusion space may be complicated when the value of $2 a$ is close to $w$. We evaluated concentration profiles of the reduced moiety by counting the number of the moiety passing at a given position and averaging them. Figure 8 shows examples of the concentration profiles for some values of $n$ or $w / 2 a$. The profile at $n=6$ has an approximately linear variation for $0<x<w$ except for the cathode. A profile for a large $n$ shows two corners at $x=2 a$ and $w-2 a$. In the domain of $0<x<2 a$, there is the anode-going translation supply of the reduced species but is no cathode-going demand. Consequently, the reduced species is accumulated in the domain of $0<x<2 a$. The accumulated amount is smoothed by the rotation to yield the linear increase profile. The accumulation causes the slope in the domain of $0<x<2 a$ larger than the slope in the domain of $2 a<x<w-2 a$.

\section{Conclusion}

The steady-state current associated with both the translational and the rotational diffusion in the thin layer cell is smaller than current controlled only by the translation. Of interest is the proportionality of the current to $n^{-1 / 2}$ for large values of $n$. This relation was applied to the ferrocene-immobilized latex particle by replacing $n$ by $a$, and was justified experimentally as effects of the diffusion-controlled current on particle size. It may be helpful for quantitative analysis of general size effects in the diagnosis whether the rotational diffusion does contribute to currents or not.

There are some limitations of the present simulation when the results are applied to real systems. The two-dimensional model does not predict accurately the real system, especially when $n$ is related with $a$. The other limitation is in the use of the thin layer cell, which is not realistic for analysis of time-dependent voltammetric data.

\section{References}


[1] C. Creutz, H. Taube, J. Am. Chem. Soc., 91 (1969) 3988.

[2] N. Hush, Prog. Inorg. Chem., 391 (1967) 8.

[3] M. B. Robin, P. Day, Adv. Inorg. Chem. Radiochem., 8 (1967) 247.

[4] R. Richardson, H. Taube, Inorg. Chem., 20 (1981) 1278.

[5] E. B. Fleischer, D. K. Lavallee, J. Am. Chem. Soc., 94 (1972) 2599.

[6] G. M. Brown, T. J. Meyer, D. O. Cowan, C. Levanda, F. Kaufman, P. V. Roling, M. D. Rausch, Inorg. Chem., 14 (1975) 506.

[7] K. Aoki, J. Chen, J. Electroanal. Chem., 380 (1995) 35.

[8] K. Aoki, J. Electroanal. Chem., 381 (1995) 185.

[9] J. Chen, K. Aoki, J. Electroanal. Chem., 402 (1996) 189.

[10] K. Aoki, J. Electroanal. Chem., 419 (1996) 33.

[11] K. Aoki, J. Chen, M. Haga, J. Electroanal. Chem., 396 (1995) 309.

[12] K. Aoki, J. Chen, H. Nishihara, T. Hirao, J. Electroanal. Chem., 416 (1996) 151.

[13] K. Aoki, H. Kamo, J. Chen, Y. Hoshino, J. Electroanal. Chem., 420 (1997) 189.

[14] H. D. Abruna in T. A. Skothrim (ED.), Electroresponsive Molecular and Polymer System, Marcel Dekker, New York, 1988, Vol. 1.

[15] J. B. Flanagan, S. M. Margel, A. J. Bard, F. C. Anson, J. Am. Chem. Soc., 100 (1978) 4248.

[16] T. Saji, N. F. Pasch, S. E. Webber, A. J. Bard, J. Phys. Chem., 82 (1978) 1101.

[17] T. W. Smith, J. E. Kuda, D. Wychick, J. Polym. Sci., 14 (1976) 2433.

[18] J. F. Hicks, D. T. Miles, R. W. Murray, J. Am. Chem. Soc., 124 (2002) 13322.

[19] K. H. Ng, H. Liu, R. M. Penner, Langmuir, 16 (2000) 4016.

[20] J. Liu, L. Cheng, Y. Song, B. Liu, S. Dong, Langmuir, 17 (2001) 6747.

[21] S. Chen, K. Huang, J. Cluster Sci., 11 (2000) 405.

[22] G. Gonzalez-Moraga, Cluster Chemistry, Spring Verlag, Berlin, 1993, pp. 54-175.

[23] C. Amatore, Y. Bouret, E. Maisonhaute, J. I. Goldsmith, H. D. Abruna, Chemphyschem, $2(2001) 130$.

[24] D. J. Diaz, G. D. Storrier, S. Bernhard, K. Takada, H. D. Abruna, Langmuir, 15 (1999) 
7351.

[25] W. Hyk, M. Karbarz, Z. Stojek, M. Ciszkowska, J. Phys. Chem. B, 108 (2004) 864.

[26] W. Zhang, I. Gaberman, M. Ciszkowska, Electroanalysis, 15 (2003) 409.

[27] W. Zhang, I. Gaberman, M. Ciszkowska, Anal. Chem., 74 (2002) 1343.

[28] C. Ma, W. Zhang, M. Ciszkowska, J. Phys. Chem. B, 105 (2001)10446.

[29] W. Hyk, M. Ciszkowska, J. Phys. Chem. B. 103 (1999) 6466.

[30] K. Aoki, Electroanalysis, 17 (2005) 1379.

[31] K. Meerholz, J. Heinze, Angew. Chem. Int. Ed. Engl. 29 (1990) 692.

[32] K. Meerholz, J. Heinze, Electrochim. Acta 41 (1995) 1839.

[33] K. Aoki., Electrochem. Comm., 2 (2000) 94.

[34] K. Aoki, J. Chen, Q. Ke, S. P. Armes, D. P. Randall, Langmuir, 19 (2003) 5511.

[35] A. Sevcik, Coll. Czech. Chem. Commun. 13 (1948) 349.

[36] R. S. Nicholson, I. Shain, Anal. Chem. 36 (1964) 706.

[37] W. H. Reinmuth, J. Am. Chem. Soc., 79 (1957) 6358.

[38] H. Matsuda, Y. Ayabe, Z. Elektrochem., 59 (1955) 494.

[39] C. Xu, K. Aoki, Langmuir, 20 (2004) 10194.

[40] J. Chen, Z. Zhang, J. Electroanal. Chem. 583 (2005) 116.

[41] C. Xu, K. Aoki, Langmuir, 20 (2004) 10194.

[42] L. Han, J. Chen, I. Ikeda, Chem. Let. 34 (2005) 1512.

[43] P. W. Atkins, Physical Chemistry, Sixth edition, Oxford University Press, Oxford, 1998, p.749.

[44] R. B. Bird, R. C. Armstrong, O. Hassager, Dynamics of Polymeric Liquids, Vol. 1. Fluid Mechanics, Chapter 1, Exmple 1.4-3., 1987, Wiley.

[45] C. Xu, J. Chen, K. Aoki, Electrochem. Commun., 5 (2003) 506.

\section{Figure Captions}

Fig. 1. Illustrative model of the cell and the polygonal particle for $n=8$. 
Fig. 2. Flow chart (left) of the key part of the Monte Carlo simulation, and an example (right) of the movement of a hexagon, where $r$ is a random number.

Fig. 3. Dependence of the square-root of the time-averaged current on $1 / N$, obtained from the Monte Carlo simulation for $n=1$ without rotation. The line has the theoretical slope, $2^{-1 / 2}$, from Eq. (5).

Fig. 4. Trajectory of an indicated side of the 80-regular polygon at $w / 2 a=1.96$ and $N=50$.

Fig. 5. Variation of the square-root of the time-averaged current on $1 / N$ for $n=2$ (open circles), 4 (full circles), 8 (open triangles), 10 (full triangles), 20 (open squares), 40 (full squares). Lines (a) and (b) have slopes, $(2 / 2)^{1 / 2}$ and $(40 / 2)^{1 / 2}$, for $n=2$ and 40 , respectively from Eq.(5).

Fig. 6. Dependence of the ratio of the simulated current to the current in Eq.(1) on logarithms of $n$ for $N=60$ (open circles), 120 (full circles) and 180 (triangles).

Fig. 7. Variation of the experimental data of $n / n_{\mathrm{UV}}$ with $a$ on the logarithmic scales, obtained from (open circles) the ferrocene-including polystyrene latex at the volumetrically uniform distribution [39] and (full circles) the chemically immobilized ferrocenyl shell on the polystyrene latex core [40].

Fig. 8. Current distribution at $(n, w / 2 a)=(a)(6,52.4)$, (b) $(20,15.7),(c)(50,6.3)$, and (d) $(90$, 3.5) for $N=100$. 


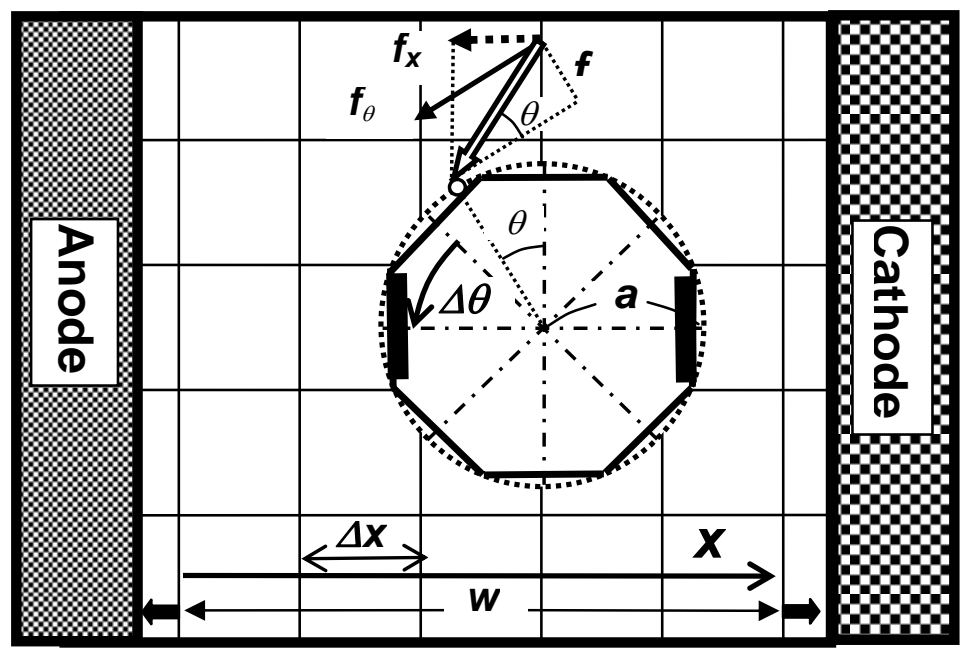

Figure 1. K. Aoki

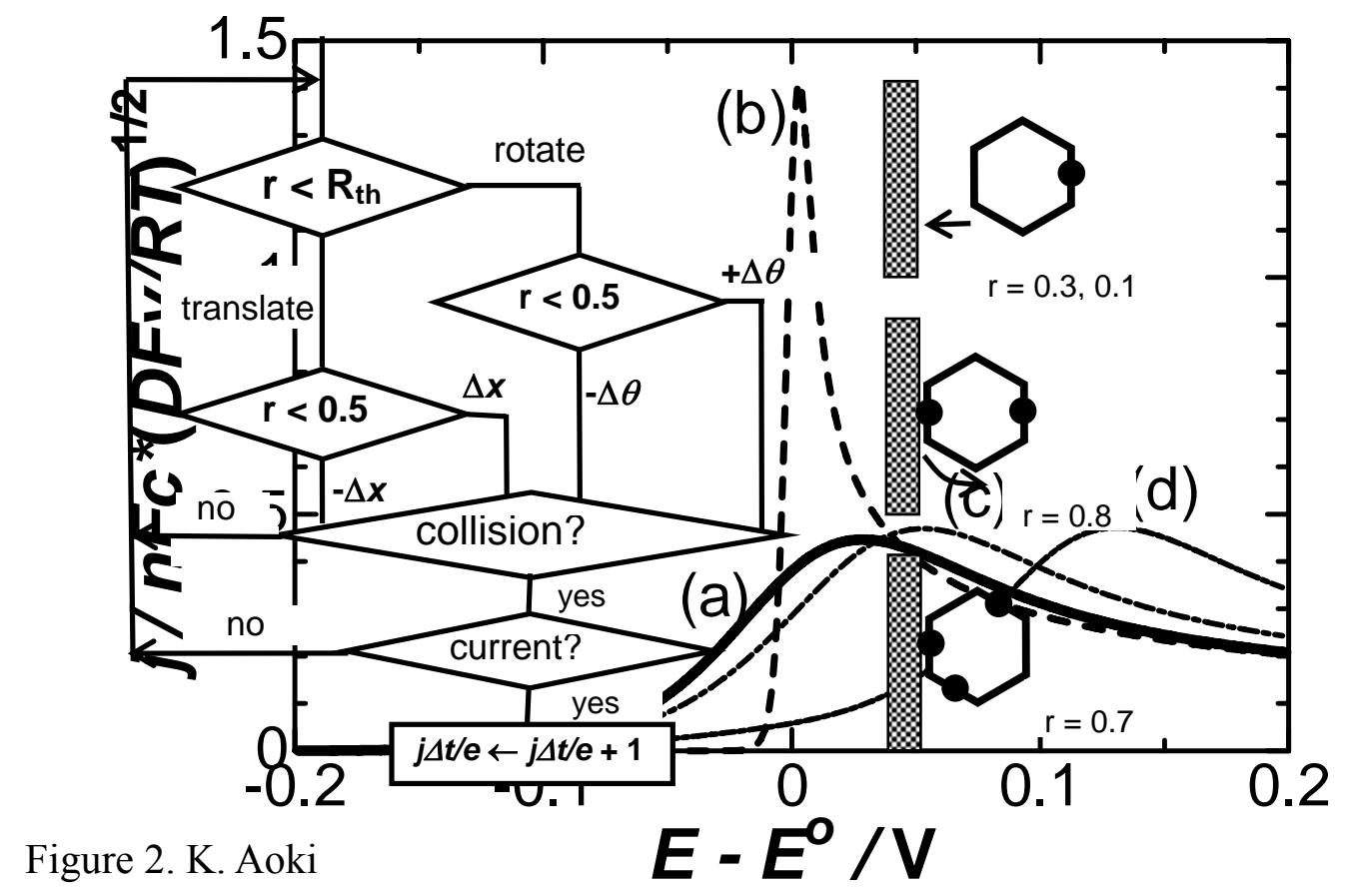




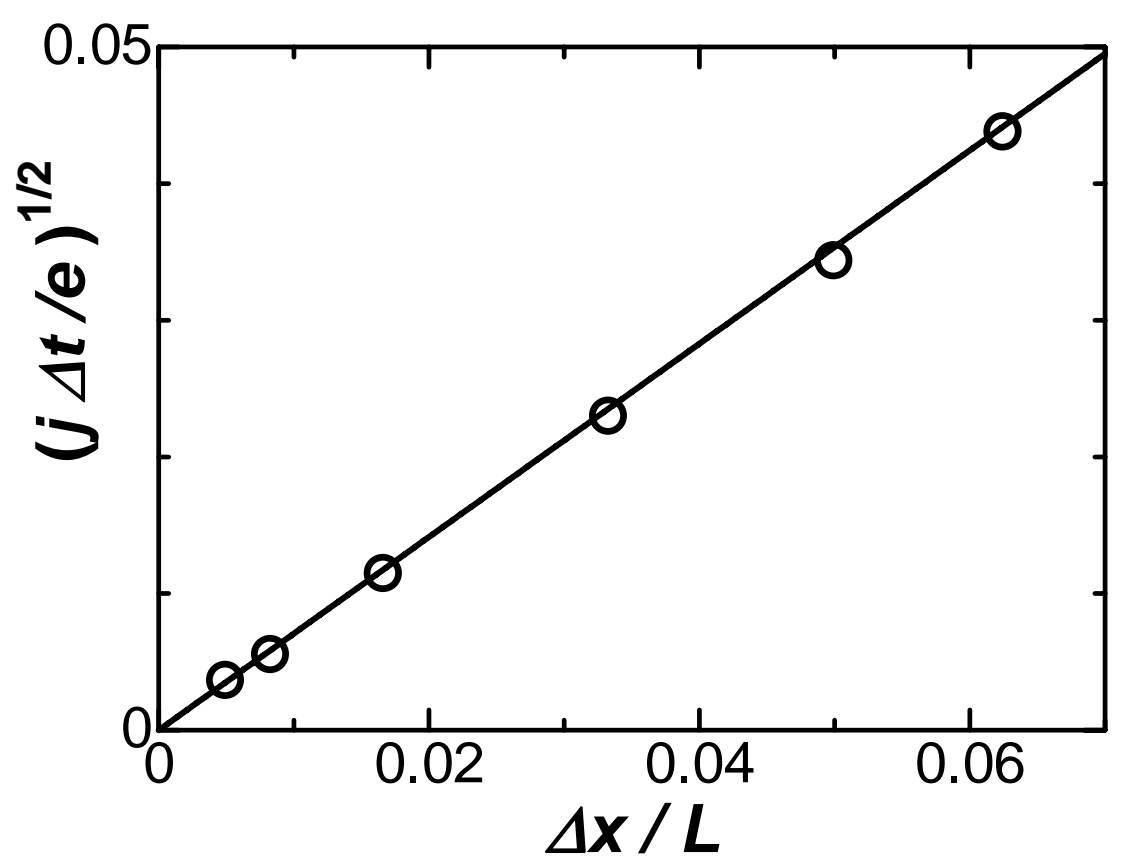

Figure 3 K. Aoki

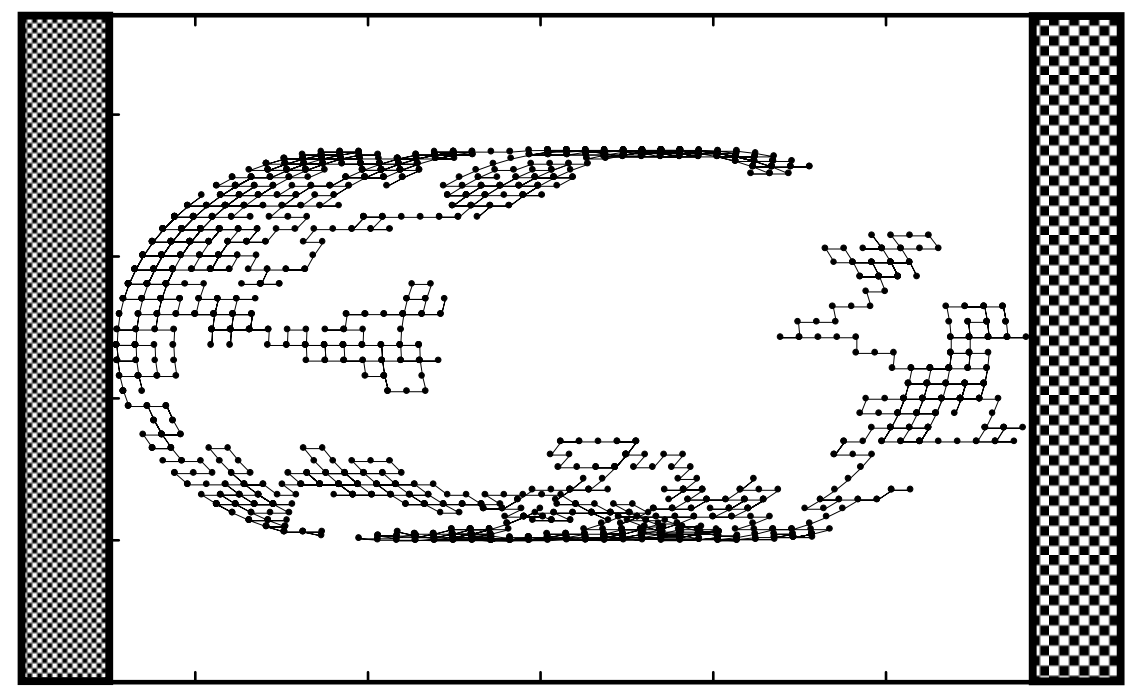

Figure 4. K. Aoki 


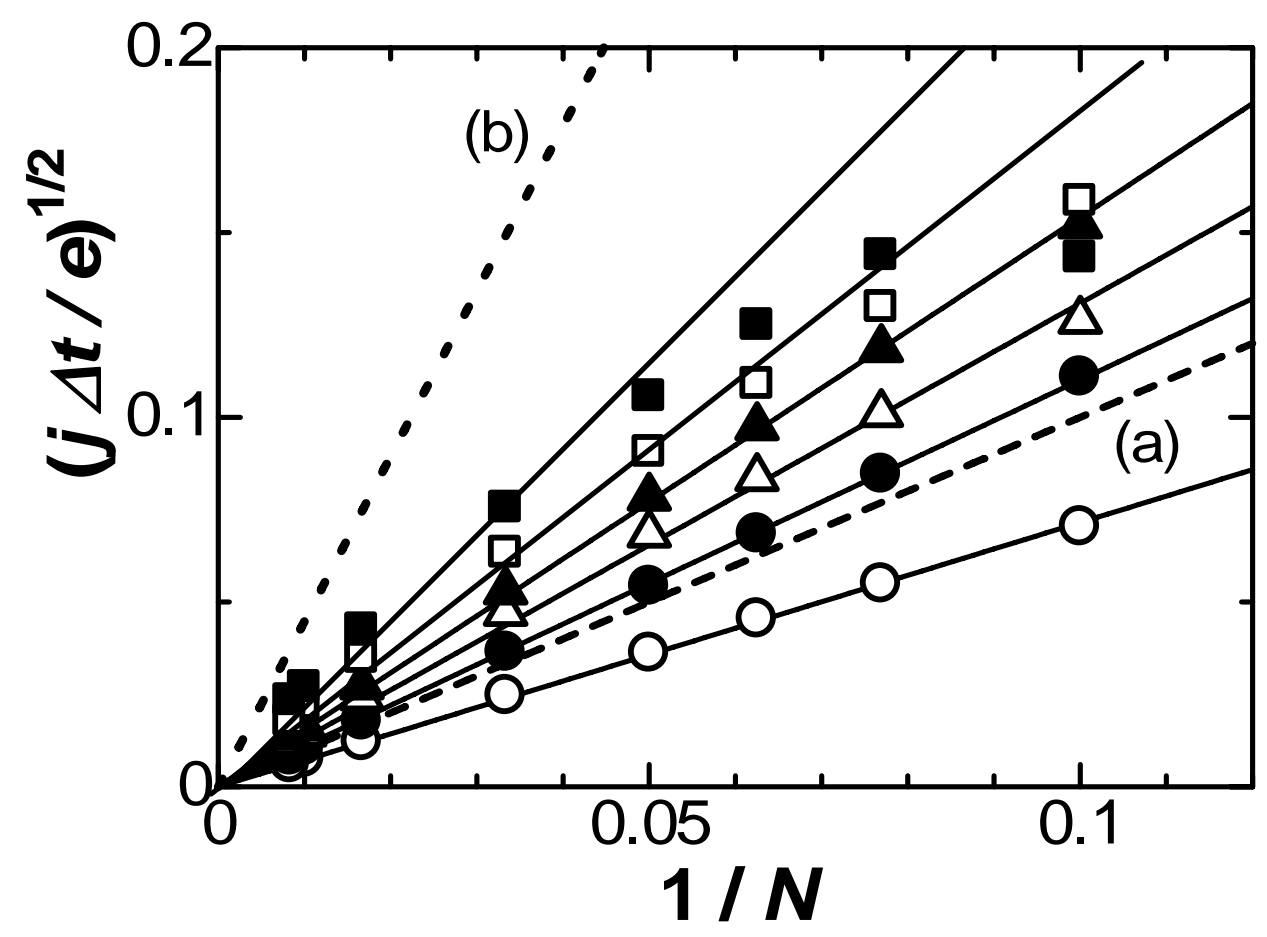

Fig. 5. K. Aoki

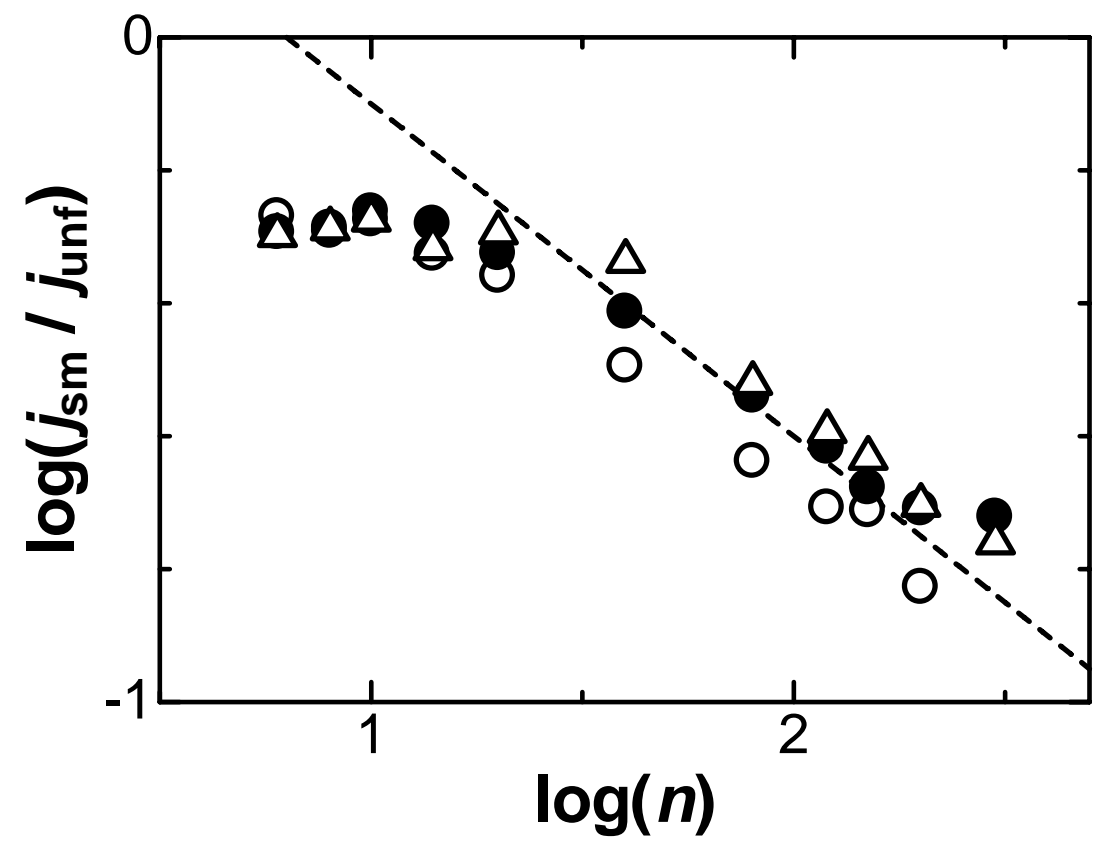

Fig 6. K. Aoki 
Fig. 7 K. Aoki
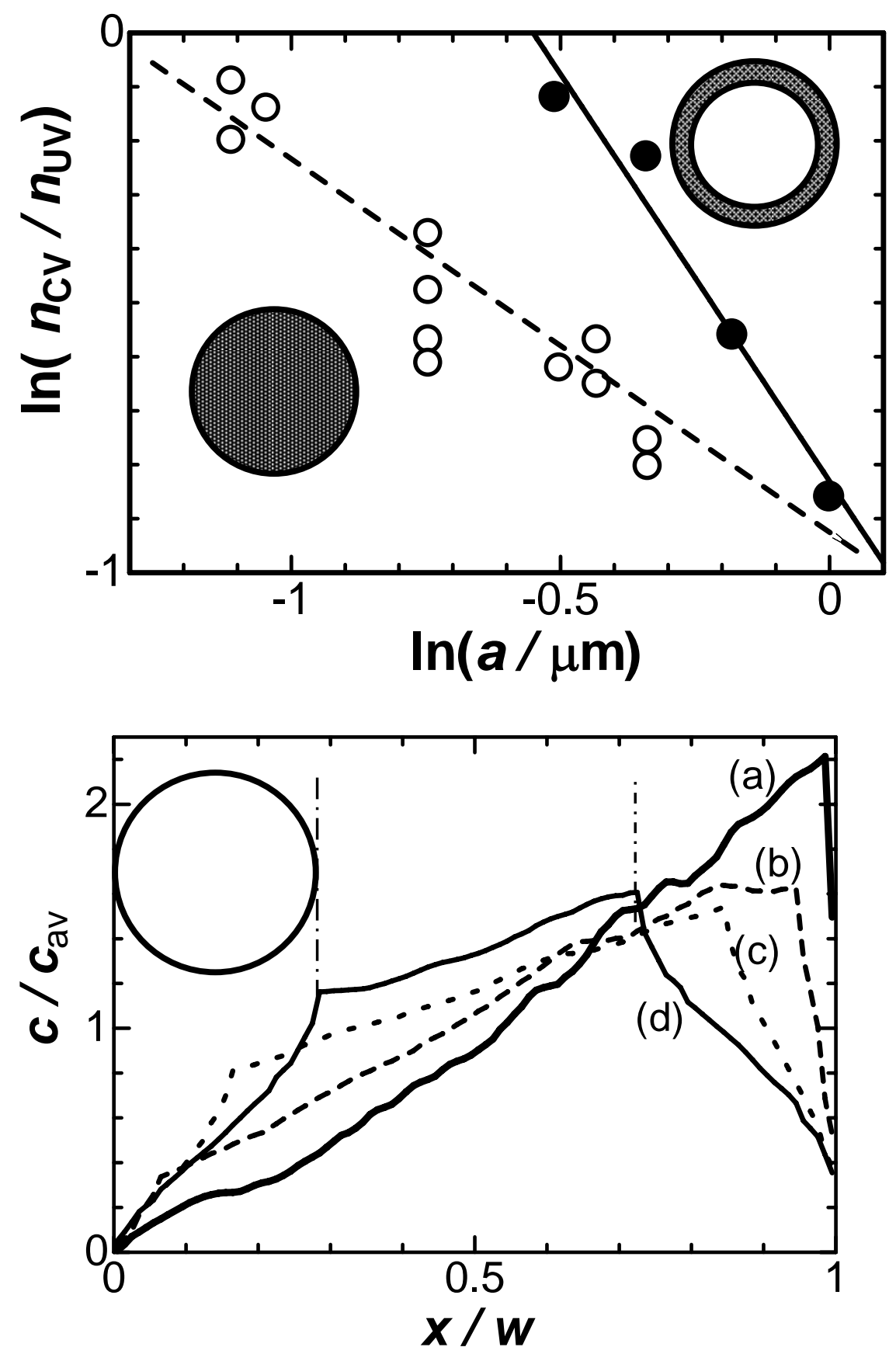

Fig. 8. K. Aoki 